Classification

Physics Abstracts

$44.00-04.00$

\title{
La pompe à chaleur à compression-absorption. Influence des différences de prix de l'électricité nuit/jour sur le coût d'exploitation
}

\author{
M. R. Jeday et P. Le Goff \\ Laboratoire des Sciences du Génie Chimique, E.N.S.I.C., 1, rue Grandville, 54042 Nancy Cedex, France
}

(Reçu le 15 décembre 1986, accepté le 12 février 1987)

\begin{abstract}
Résumé. - La Pompe A Chaleur à Compression-Absorption à Fonctionnement Alterné (ou PACCAFA) est un nouveau type de pompe à chaleur décrit précédemment (Réf. [1]), intermédiaire entre la PAC à compression et la PAC à absorption. Par comparaison à une PAC à compression classique, la PACCAFA consomme davantage d'électricité en heures creuses et moins en heures de pointe grâce à un système de stockage d'énergie sous forme de séparation-mélangeage d'un couple solvant-soluté. Dans l'exemple étudié, il s'agit d'ammoniac et de thiocyanate de sodium. Nous déterminons dans quelles conditions la valeur moyenne sur 24 heures du coût d'exploitation énergétique d'une telle PACCAFA serait inférieure à celle d'une PAC à compression classique. Ces conditions sont exprimées sous deux formes : - la valeur minimale du rapport des prix de l'électricité en heures pleines et en heures creuses; - la durée minimale de la période " heures creuses » pendant une journée de 24 heures.
\end{abstract}

\begin{abstract}
The Compression-Absorption Heat Pump Alternatively Working (CAHPAW) is a new type of heat pump, described previously (Ref. [1]) and is midway between the Compression heat pump and the Absorption heat pump. Compared to a standard compression heat pump, the CAHPAW consumes more electricity during off-peak hours and less during peak hours thanks to an energy storage system in the form of the separation-mixing of a solvent-solution pair. In the example studied, ammonia and thiocyanate of sodium were used. We determine under what conditions the average energy exploitation cost during a period of 24 hours for such a CAHPAW would be smaller than that of a standard compression heat pump. These conditions can be expressed in two forms : - the minimum ratio of electricity prices during peak and off-peak hours ; - the minimum duration of an « off-peak period» during a 24 hour day.
\end{abstract}

\section{Notations}

$C$ Coût d'exploitation énergétique (en $\mathrm{FF} /$ jour)

$\Delta C$ Surcoût d'exploitation énergétique (en $\mathrm{FF} / \mathrm{jour}$ )

CON Contenu énergétique de la solution (en $\mathrm{kJ} / \mathrm{kg}$ )

COP Coefficient de performance

$H \quad$ Enthalpie massique (en $\mathrm{kJ} / \mathrm{kg}$ )

$k \quad$ Coefficient de proportionnalité dans l'expression du prix unitaire de l'énergie

$\dot{M}_{\mathrm{c}} \quad$ Débit massique du concentrat (en $\mathrm{kg} / \mathrm{s}$ )

$\dot{M}_{\mathrm{d}} \quad$ Débit massique du diluat (en $\mathrm{kg} / \mathrm{s}$ )

$\dot{M}_{\mathrm{v}} \quad$ Débit massique du solvant (en $\mathrm{kg} / \mathrm{s}$ )

$\dot{Q}_{\mathrm{u}} \quad$ Puissance thermique utile (en $\mathrm{kW}$ )

$t \quad$ Durée opératoire d'un régime donné (en heures)

$v \quad$ Prix unitaire de l'énergie électrique (en $\mathrm{FF} / \mathrm{kWh}$ )

$\dot{W} \quad$ Puissance mécanique fournie au compresseur (en $\mathrm{kW}$ )

$x_{\mathrm{c}} \quad$ Titre massique du concentrat (en $\mathrm{kg}$ de $\mathrm{NaSCN} / \mathrm{kg}$ solution)

$x_{\mathrm{d}} \quad$ Titre massique du diluat (en $\mathrm{kg}$ de $\mathrm{NaSCN} / \mathrm{kg}$ solution) 


\section{Indices}

m mélangeage

s séparation

c périodes creuses

$\mathrm{p}$ périodes pleines

\section{Introduction.}

Dans une publication précédente (Réf. [1]), nous avons proposé un nouveau type de pompe à chaleur qui est, par certains aspects, intermédiaire entre la $\mathrm{PAC}$ à absorption et la PAC à compression. Comme la PAC à absorption, elle utilise un fluide de travail qui est un mélange binaire d'un solvant volatil et d'un soluté non volatil. Comme la PAC à compression, elle utilise de l'énergie mécanique comme énergie de haut niveau. De plus cette PAC a la particularité d'être composée d'un seul et même appareil qui joue le rôle alternativement du mélangeur et du séparateur thermomécaniques : d'où vient son nom de PACCAFA pour Pompe A Chaleur à Compression-Absorption à Fonctionnement Alterné.

Comme une PAC à compression, la PACCAFA est formée d'un évaporateur, d'un compresseur, d'un condenseur et d'un détendeur auxquels on a ajouté trois réservoirs de stockage respectivement pour le solvant, la solution concentrée et la solution diluée (voir Fig. 1).
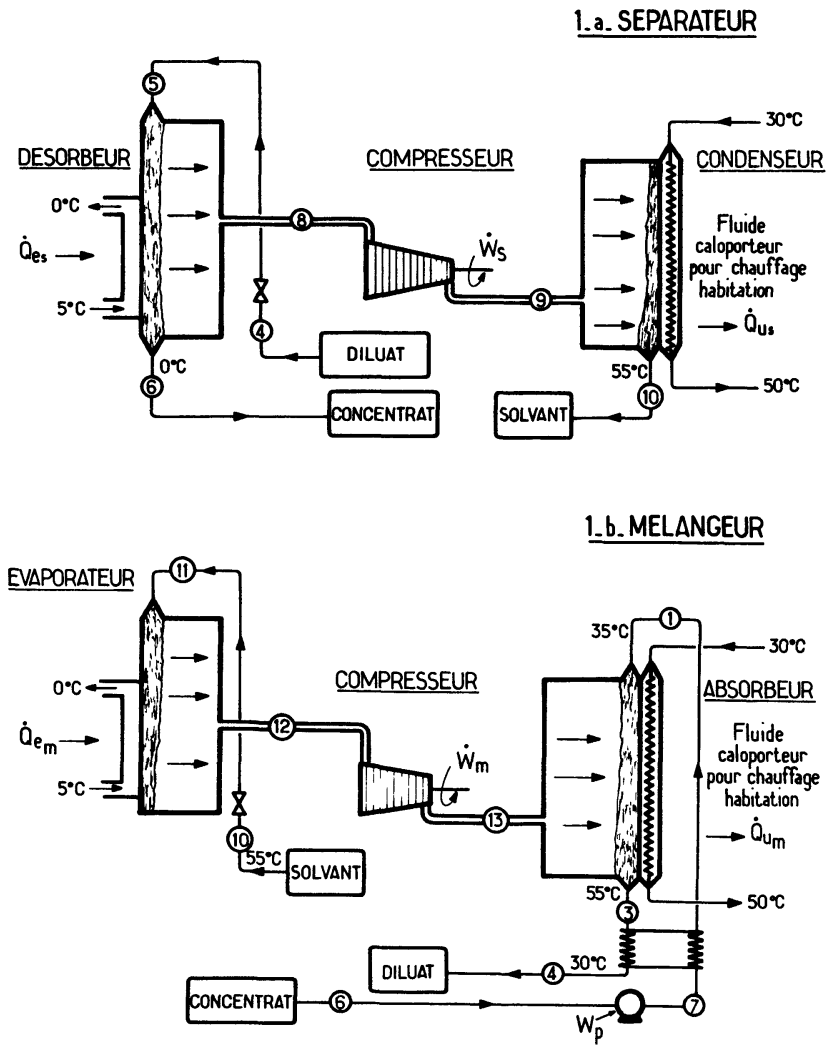

Fig. 1. - Schéma de principe de la PACCAFA.

[Principle of the CAHPAW.]

\section{Avantages de la PACCAFA.}

Rappelons que dans une pompe à chaleur à compression classique, le coefficient de performance (COP) est d'autant plus petit que la différence de température entre évaporateur et condenseur est plus grande. Ceci est particulièrement gênant pour le chauffage des bâtiments dans les régions froides.

Dans le cas extrême où l'on veut produire de l'eau chaude à $80^{\circ} \mathrm{C}$, avec une température extérieure inférieure à $0^{\circ} \mathrm{C}$, le COP devient si faible que le système n'est plus rentable. C'est pourquoi on considère généralement qu'une telle $\mathrm{PAC}$ classique air/eau ne peut pas fonctionner seule. Il faut nécessairement lui associer une chaudière d'appoint, à combustible, pour les périodes les plus froides. Un tel surinvestissement constitue un important inconvénient de ce système bi-énergie.

Le système PACCAFA a au contraire l'avantage d'être «mono-énergie " en ce sens qu'il fonctionne uniquement à l'électricité, même par les temps les plus froids et ne nécessite pas l'installation d'une chaudière d'appoint. Son coût d'investissement est donc plus faible.

De plus la présence des réservoirs de stockage (qui constituent un stockage d'énergie sous forme physico-chimique de séparation-mélangeage), permet de rendre indépendantes dans le temps, les opérations de consommation d'énergie mécanique (donc d'électricité) et de production de chaleur.

Ainsi ce procédé de stockage pourra mettre à profit les modulations du tarif d'électricité proposé par E.D.F. pour les heures creuses de nuit et même de fin de semaine, et inversement, le déstockage permettra de fournir une surpuissance thermique quand la demande de chauffage est très grande, pendant les jours froids.

\section{Rappel du principe de la PACCAFA.}

2.1 SCHÉMA DE PRINCIPE. - Partons d'une PAC à compression classique qui fonctionne avec un fluide volatil pur (par exemple : l'ammoniac). Remplaçons ce fluide pur par un mélange binaire composé de ce même fluide appelé «solvant volatil » et d'un soluté non volatil qui a la propriété d'abaisser fortement la pression de vapeur de solvant (par exemple : le thiocyanate de sodium dans le cas de l'ammoniac).

L'ensemble Evaporateur-Compresseur-Condenseur fonctionne alors en régime cyclique tantôt en « séparateur» du mélange, tantôt en «mélangeur » des constituants du mélange.

Nous avons repéré par des nombres :

de 1 à 7 , les états successifs de la solution, de 8 à 13, les états successifs du solvant pur.

Le tableau I donne la signification de ces nombres.

* Quand l'ensemble opère en « séparateur », une solution diluée ou « diluat » (point 5) est injectée en 
tête de l'évaporateur et on collecte une solution concentrée ou «concentrat» (point 6) en pied de l'appareil, alors que le solvant pur est condensé et recueilli au condenseur (point 10) (cf. Fig. 1a).

Tableau I.

\section{Repères du circuit de solution}

1. Concentrat sortant réchauffé de l'échangeur et entrant dans l'absorbeur.

2. Diluat liquide saturé sortant de l'absorbeur.

3. Diluat refroidi sortant de l'échangeur thermique.

4. Diluat sortant du détendeur et entrant dans le désorbeur.

5. Concentrat sortant du désorbeur à l'état liquide saturé.

6. Concentrat sortant de la pompe et entrant dans l'échangeur thermique.

\section{Repères du circuit de solvant pur}

7. Vapeur surchauffée sortant du désorbeur.

8. Vapeur surchauffée sortant du compresseur en position séparation.

9. Liquide saturé sortant du condenseur.

11. Mélange liquide-vapeur sortant du détendeur et entrant dans l'évaporateur.

12. Vapeur saturée sortant de l'évaporateur.

13. Vapeur surchauffée sortant du compresseur en position mélangeage.

* Quand il opère en "mélangeur », on injecte au contraire du solvant pur (point 11) en tête de l'évaporateur et du concentrat (point 1) en tête du condenseur. La vapeur de solvant (point 13) se condense et se mélange dans le concentrat pour former le diluat (point 3) (cf. Fig. 1b).

Trois réservoirs, respectivement pour le concentrat, le diluat et le solvant pur, sont alternativement remplis et vidés au cours de chaque cycle de séparation-mélangeage.

2.2 REPRÉSENTATION GRAPHIQUE DE LA SÉPARATION ET DE MÉLANGEAGE. - Les figures 2 et 3 montrent - d'une part, sur le diagramme de Ponchon $(H, x)$, les opérations d'absorption et de désorption - d'autre part, sur le second diagramme de Mollier $(\operatorname{Ln} P, H)$, le tracé des parcours suivis par le solvant pur dans les deux sous-ensembles de séparation et de mélangeage.

Les points (A) et (S) désignent respectivement les résultats de l'absorption et de la désorption.

En calculant les bilans de matière et d'enthalpie dans l'absorbeur, on démontre aisément que le résultat du mélangeage de (1) et (13) est représenté par un point (A) défini par l'addition vectorielle :

$$
\dot{M}_{\mathrm{v}}(\mathbf{A}, 13)+\dot{M}_{\mathrm{c}}(\mathbf{A}, \mathbf{1})=0 .
$$

C'est la classique « règle des leviers».

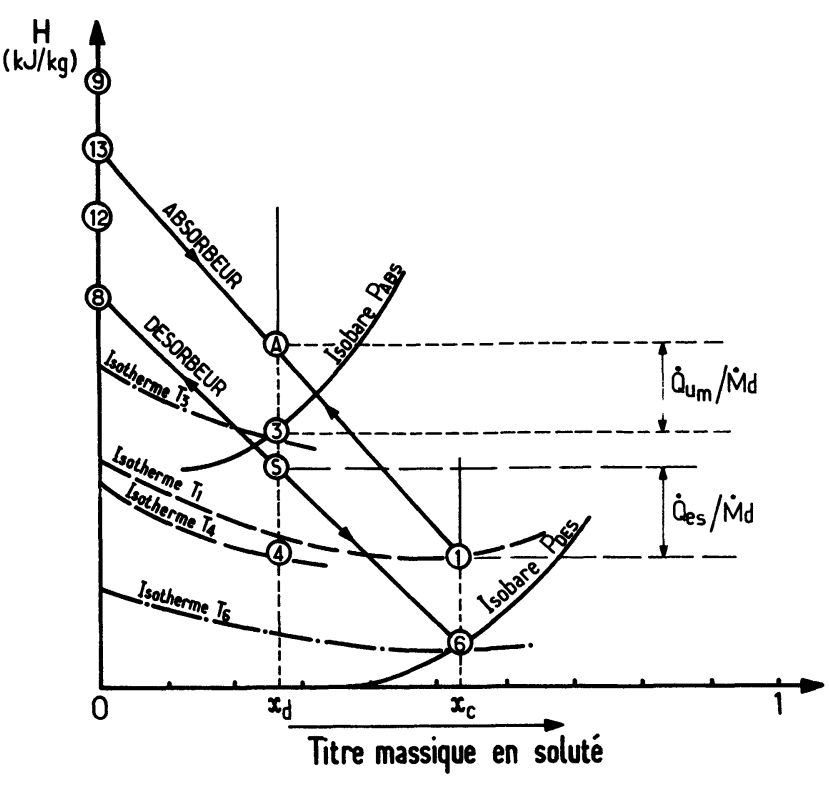

Fig. 2. - Diagramme de Ponchon : représentation de deux sous-ensembles de séparation et de mélangeage par addition des vecteurs.

[Ponchon's diagram : representation of the two sets of separation and mixing with addition of vectors.]

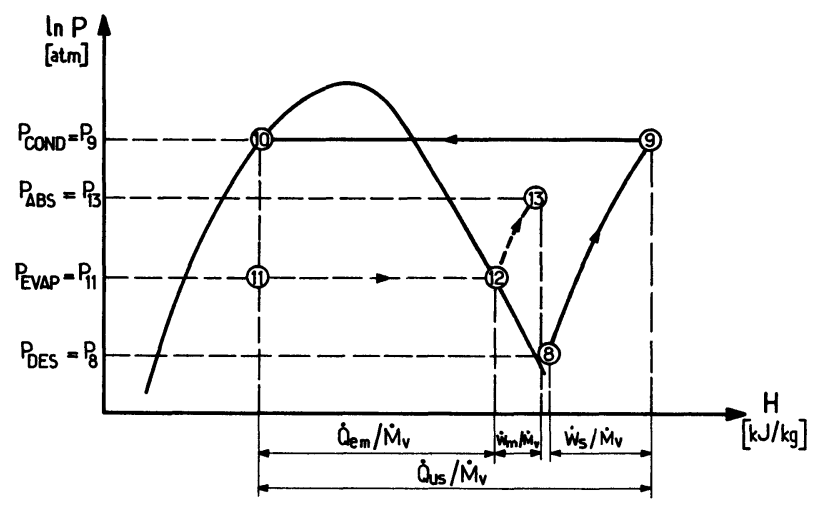

Fig. 3. - Second diagramme de Mollier: tracé des parcours suivis par le solvant dans les deux sous-ensembles — sous-ensemble de Séparation --- sous-ensemble de Mélangeage.

[Mollier's second diagram : Operating line of the solvent in the two sets. _- Separation set ; --- Mixing set.]

La différence des ordonnées des points (A) et (3) mesure directement le flux de chaleur $\dot{Q}_{\mathrm{u}}$ rapporté à la masse de diluat :

$$
|\mathrm{A}, 3|=\frac{\dot{Q}_{\mathrm{um}}}{\dot{M}_{\mathrm{d}}} .
$$

En calculant de même les bilans de matière et d'enthalpie dans le désorbeur, on montre que le résultat de la séparation du diluat en (6) et (8), 
représenté par le point (S), est défini par l'addition vectorielle :

$$
\dot{M}_{\mathrm{v}}(\mathbf{S}, \mathbf{8})+\dot{M}_{\mathrm{c}}(\mathrm{S}, \mathbf{6})=0
$$

et la différence des ordonnées des points (S) et (4) donne le flux de chaleur $\dot{Q}_{\text {es }}$ rapporté à la masse de diluat :

$$
|\mathrm{S}, 4|=\frac{\dot{Q}_{\mathrm{es}}}{\dot{M}_{\mathrm{d}}}
$$

En outre les différences des abscisses des 6 points, présentés sur le second diagramme de Mollier, mesurent directement les flux de chaleur et de travail rapportés à la masse de solvant, soit :

$$
\begin{gathered}
|9,8|=\frac{\dot{W}_{\mathrm{s}}}{\dot{M}_{\mathrm{v}}} \quad|9,10|=\frac{\dot{Q}_{\mathrm{us}}}{\dot{M}_{\mathrm{v}}} \\
|12,11|=\frac{\dot{Q}_{\mathrm{em}}}{\dot{M}_{\mathrm{v}}} \quad|13,12|=\frac{\dot{W}_{\mathrm{m}}}{\dot{M}_{\mathrm{v}}} .
\end{gathered}
$$

2.3 LES DEUX CRITÈRES DE QUALITÉ COP ET CON. - Pour caractériser la qualité d'une pompe à chaleur, on utilise habituellement le « coefficient de performance » (COP) dans sa définition générale, soit le rapport de la chaleur utile produite à la somme des énergies coûteuses consommées. L'expression du COP (calculée en Réf. [2]) et exprimée à l'aide des notations de la figure 1 , est la suivante :

$$
\mathrm{COP}=\frac{\left(H_{13}+H_{9}-H_{10}\right)+\frac{x_{\mathrm{d}} H_{1}-x_{\mathrm{c}} H_{3}}{x_{\mathrm{c}}-x_{\mathrm{d}}}}{\left(H_{13}-H_{12}\right)+\left(H_{9}-H_{8}\right)+\frac{x_{\mathrm{d}}}{x_{\mathrm{c}}-x_{\mathrm{d}}}\left(H_{7}-H_{6}\right)}
$$

Nous avons d'autre part insisté précédemment (Réf. [3]) sur la nécessité de prendre en compte un autre critère : le «contenu énergétique de la solution » (CON) qui est égal au rapport de la chaleur utile produite à la masse totale de solution diluée en circulation. En écrivant les bilans de matière et d'enthalpie au cours d'un cycle complet de stockagedéstockage, on parvient aisément à l'expression suivante :

$$
\begin{aligned}
\operatorname{CON}= & \left(\frac{x_{\mathrm{c}}-x_{\mathrm{d}}}{x_{\mathrm{c}}}\right) \times \\
& \times\left(H_{13}+H_{9}-H_{10}+\frac{x_{\mathrm{d}} H_{1}-x_{\mathrm{c}} H_{3}}{x_{\mathrm{c}}-x_{\mathrm{d}}}\right) .
\end{aligned}
$$

\section{Expressions du coût d'exploitation énergétique.}

Afin de comparer le système PACCAFA (décrit aux $\S 21$ et 22) à une PAC à compression utilisée dans les mêmes conditions (mêmes températures, puissance et fluide de travail), nous évaluons le coût d'exploitation énergétique pour chaque cas selon les expressions données ci-dessous :

3.1 PAC A COMPRESSION ClASSIQUE. - Nous distinguerons deux composantes dans le coût total d'exploitation énergétique $C_{1}$, soit :

$$
\begin{aligned}
& \left(\begin{array}{c}
\text { Coût total } \\
\text { d'exploitation } \\
\text { énergétique }
\end{array}\right)=\left(\begin{array}{c}
\text { Coût } \\
\text { d'exploitation } \\
\text { en heures creuses }
\end{array}\right)+\left(\begin{array}{c}
\text { Coût } \\
\text { d'exploitation } \\
\text { en heures pleines }
\end{array}\right) \\
& C_{1}=C_{\mathrm{c}}+C_{\mathrm{p}}
\end{aligned}
$$

avec

$$
\begin{aligned}
& C_{\mathrm{c}}=\dot{W} \cdot t_{\mathrm{c}} \cdot v_{\mathrm{c}} \\
& C_{p}=\dot{W} \cdot t_{p} \cdot v_{p}
\end{aligned}
$$

Dans ces 2 relations :

$\dot{W}$ est la puissance mécanique fournie au compresseur (en $\mathrm{kW})$,

$v$ est le prix unitaire de l'énergie (en $\mathrm{FF} / \mathrm{kWh}$ ), $t$ est la durée de fonctionnement (en heures).

Les indices $c$ et $p$ désignent respectivement heures creuses $\left(t_{\mathrm{c}}=8\right.$ heures/jour $)$ et heures pleines $\left(t_{\mathrm{p}}=16\right.$ heures/jour).

La relation (8) devient :

$$
C_{1}=\dot{W} \cdot\left[t_{\mathrm{c}} \cdot v_{\mathrm{c}}+t_{\mathrm{p}} \cdot v_{\mathrm{p}}\right]
$$


3.2 LA PACCAFA. - La figure 4 donne la puissance consommée au cours d'une journée de 24 heures par le système PACCAFA où la période de séparation, de durée $t_{\mathrm{s}}$, est supérieure à la durée $t_{\mathrm{c}}$ des heures creuses.

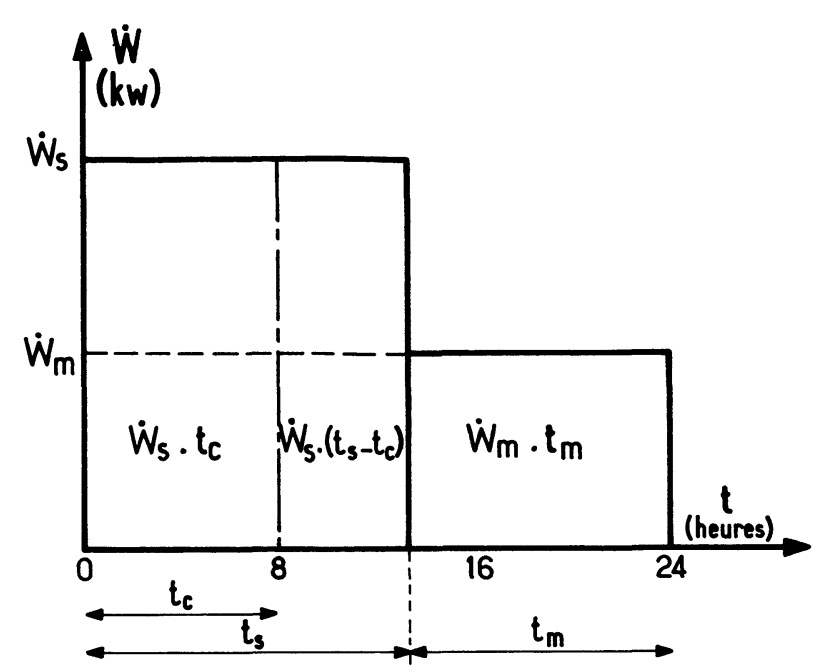

Fig. 4. - Consommation de l'énergie électrique en fonction de la durée de fonctionnement.

[Electricity consumption as a function of the working duration.]

Le coût total d'exploitation énergétique de la PACCAFA, $C_{2}$, est la somme de trois termes :

$$
C_{2}=C_{\mathrm{m}}+C_{\mathrm{s}}^{\prime}+C_{\mathrm{s}}^{\prime \prime} \text {. }
$$

Dans cette expression :

$C_{\mathrm{m}}$ est le coût d'exploitation en période de mélangeage de durée $t_{\mathrm{m}}<t_{\mathrm{p}}=16$ heures,

$C_{\mathrm{s}}^{\prime}$ est le coût d'exploitation en période de séparation de durée $t_{\mathrm{c}}=8$ heures,

$C_{\mathrm{s}}^{\prime \prime}$ est le coût d'exploitation en période de séparation de durée $\left(t_{\mathrm{s}}-8\right)$ heures.

Les 3 coûts $\left(C_{\mathrm{m}}, C_{\mathrm{s}}^{\prime}\right.$ et $\left.C_{\mathrm{s}}^{\prime \prime}\right)$ sont calculés à partir des relations suivantes :

$$
\left\{\begin{array}{l}
C_{\mathrm{m}}=\dot{W}_{\mathrm{m}} \cdot t_{\mathrm{m}} \cdot v_{\mathrm{p}} \\
C_{\mathrm{s}}^{\prime}=\dot{W}_{\mathrm{s}} \cdot t_{\mathrm{c}} \cdot v_{\mathrm{c}} \\
C_{\mathrm{s}}^{\prime \prime}=\dot{W}_{\mathrm{s}} \cdot\left(t_{\mathrm{s}}-t_{\mathrm{c}}\right) \cdot v_{\mathrm{p}}
\end{array}\right.
$$

En combinant les expressions respectives (10) et (11), nous obtenons :

$C_{2}=\dot{W}_{\mathrm{m}} \cdot t_{\mathrm{m}} \cdot v_{\mathrm{p}}+\dot{W}_{\mathrm{s}} \cdot\left[t_{\mathrm{c}} \cdot v_{\mathrm{c}}+\left(t_{\mathrm{s}}-t_{\mathrm{c}}\right) \cdot v_{\mathrm{p}}\right]$.

Parallèlement, nous définissons le surcoût d'exploitation énergétique $\Delta C$ par la différence :

$$
\Delta C=C_{2}-C_{1}
$$

que l'on peut mettre encore sous cette forme :

$$
\begin{aligned}
\Delta C=\left[\dot{W}_{\mathrm{s}}-\dot{W}\right] \cdot t_{\mathrm{c}} \cdot v_{\mathrm{c}}+ \\
\quad+\left[\dot{W}_{\mathrm{m}} t_{\mathrm{m}}+\dot{W}_{\mathrm{s}}\left(t_{\mathrm{s}}-t_{\mathrm{c}}\right)-\dot{W} t_{\mathrm{p}}\right] \cdot v_{\mathrm{p}} .
\end{aligned}
$$

Nota : Pour les périodes de séparation de durée $t_{\mathrm{s}}$ inférieure à celle des heures creuses $\left(t_{\mathrm{c}}=8\right.$ heures), le coût d'exploitation énergétique $C_{2}$ est donné par l'expression:

$C_{2}=\dot{W}_{\mathrm{s}} \cdot t_{\mathrm{s}} \cdot v_{\mathrm{c}}+\dot{W}_{\mathrm{m}} \cdot\left[\left(t_{\mathrm{c}}-t_{\mathrm{s}}\right) \cdot v_{\mathrm{c}}+t_{\mathrm{p}} \cdot v_{\mathrm{p}}\right]$.

\section{Un exemple concret : le chauffage d'un bâtiment.}

Afin de comparer quantitativement la PACCAFA et une PAC classique, dans les mêmes conditions d'utilisation, nous avons effectué tous les calculs dans un cas concret : celui d'un bâtiment standard moyen qui doit être maintenu à une température constante (par exemple $+19{ }^{\circ} \mathrm{C}$ ) dans un environnement à température également constante (par exemple $+5{ }^{\circ} \mathrm{C}$ ). Il faut donc lui fournir une puissance de chauffe constante, 24 heures sur 24 (par exemple $10 \mathrm{~kW})$.

Nous supposerons que le chauffage du bâtiment se fait par circulation d'eau chaude, qui revient à $30^{\circ} \mathrm{C}$ et que la pompe à chaleur doit remonter à $50^{\circ} \mathrm{C}$. La source de chaleur froide, utilisée par la pompe à chaleur est l'air extérieur, disponible à $+5{ }^{\circ} \mathrm{C}$ et restitué à $0{ }^{\circ} \mathrm{C}$.

Nous supposerons un pincement uniforme de $5^{\circ} \mathrm{C}$ dans tous les échangeurs (évaporateurs et absorbeurs-condenseurs). Ainsi la surface du liquide en cours d'évaporation dans l'évaporateur aura son point le plus froid à $-5{ }^{\circ} \mathrm{C}$, tandis que la surface condensante dans l'absorbeur-condenseur, aura son point le plus chaud à $+55^{\circ} \mathrm{C}$.

Nous prendrons comme prix de l'électricité un tarif moyen proposé par E.D.F. (Réf. [7]), soit :

$v_{\mathrm{p}}=0,5037 \mathrm{FF} / \mathrm{kWh}$ en heures pleines, soit $t_{\mathrm{p}}=16$ heures par jour,

$v_{\mathrm{c}}=0,2881 \mathrm{FF} / \mathrm{kWh}$ en heures creuses, soit $t_{\mathrm{c}}=8$ heures par jour.

\subsection{LA PAC A COMPRESSION CLASSIQUE COMME} RÉFÉRENCE. - Les figures 5 et 6 montrent respectivement :

* le schéma de principe d'une PAC à compression classique,

* et le tracé du cycle suivi par le solvant dans cette même machine (Réf. [4]).

* Dans l'évaporateur, à $-5^{\circ} \mathrm{C}$, l'ammoniac pur s'évapore sous une pression de 3,6 bars. Son enthalpie est $H_{16}=1256 \mathrm{~kJ} / \mathrm{kg}$.

* Dans le condenseur, à $+55^{\circ} \mathrm{C}$, l'ammoniac pur 


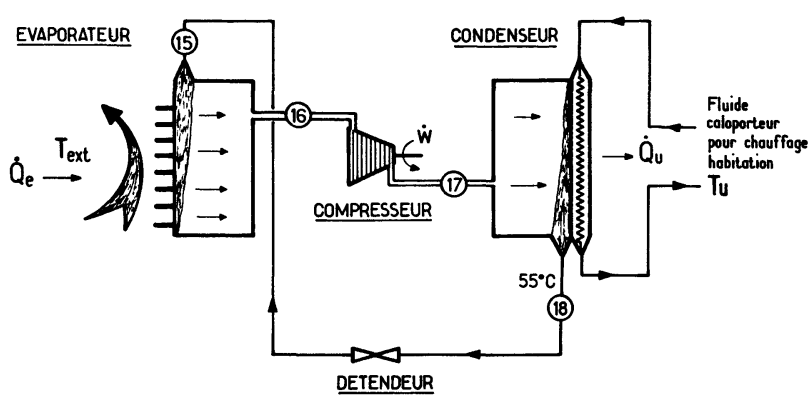

Fig. 5. - Schéma de principe d'une PAC à compression classique.

[Principle of the standard Compression heat Pump.]

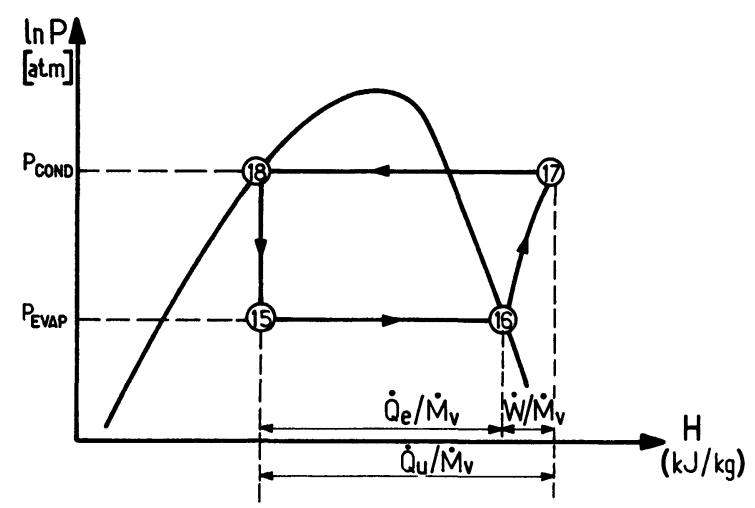

Fig. 6. - Second diagramme de Mollier : tracé du cycle suivi par le solvant.

[Mollier's second diagram : operating line followed by the solvent.]

se condense sous une pression de 23,6 bars. Son enthalpie est :

à l'état vapeur surchauffée : $H_{17}=1532 \mathrm{~kJ} / \mathrm{kg}$

à l'état liquide saturé : $H_{18}=265 \mathrm{~kJ} / \mathrm{kg}$.

Connaissant la puissance de chauffe à fournir, soit $\dot{Q}_{\mathrm{u}}=10 \mathrm{~kW}$, nous en déduisons le débit d'ammoniac à vaporiser et condenser $\dot{M}_{\mathrm{v}}$ puis la puissance mécanique à fournir au compresseur $\dot{W}$, soit :

$$
\dot{M}_{\mathrm{v}}=\frac{\dot{Q}_{\mathrm{u}}}{H_{17}-H_{18}}=0,0079 \mathrm{~kg} / \mathrm{s}
$$

$\dot{W}=\dot{M}_{\mathrm{v}} \cdot\left(H_{17}-H_{16}\right)=\frac{H_{17}-H_{16}}{H_{17}-H_{18}} \cdot \dot{Q}_{\mathrm{u}}=2,18 \mathrm{~kW}$.

Le coefficient de performance est alors :

$$
\mathrm{COP}=\frac{\dot{Q}_{\mathrm{u}}}{\dot{W}}=4,58
$$

Le coût d'exploitation énergétique sur 24 heures est :

$$
\begin{aligned}
C_{1}=\dot{W}(16 \times 0,5037+8 \times 0,2881) & = \\
& =22,59 \mathrm{FF} / \text { jour } .
\end{aligned}
$$

4.2 UN EXEMPLE DE PACCAFA. - Nous traiterons un exemple particulier où les deux solutions sont très concentrées et peu différentes, soit :

* le titre en soluté dans le concentrat : $x_{\mathrm{c}}=0,61$;

* le titre en soluté dans le diluat : $x_{\mathrm{d}}=0,54$.

En régime de mélangeage, la température de condensation de $55^{\circ} \mathrm{C}$ est celle qui correspond au diluat, la pression correspondante est 7 bars (point 3). Il suffit donc de comprimer la vapeur de 3,6 à 7 bars (au lieu de 23,6 bars), soit un taux de compression de 1,94 au lieu de 6,55.

Pour la même puissance de chauffe, soit $\dot{Q}_{\mathrm{um}}=10 \mathrm{~kW}$, le débit d'ammoniac à l'absorbeur est :

$$
\dot{M}_{\mathrm{vm}}=\frac{\dot{Q}_{\mathrm{um}}}{\left(H_{13}+\frac{x_{\mathrm{d}} H_{1}-x_{\mathrm{c}} H_{3}}{x_{\mathrm{c}}-x_{\mathrm{d}}}\right)}=0,0099 \mathrm{~kg} / \mathrm{s}
$$

et la puissance mécanique à fournir au compresseur (à un seul étage) est :

$$
\dot{W}_{\mathrm{m}}=\dot{M}_{\mathrm{vm}}\left(H_{13}-H_{12}\right)=0,87 \mathrm{~kW}
$$

soit un coefficient de performance :

$$
\mathrm{COP}_{\mathrm{m}}=\frac{\dot{Q}_{\mathrm{um}}}{\dot{W}_{\mathrm{m}}}=11,5 .
$$

En régime de séparation, le diluat s'évapore à $-5{ }^{\circ} \mathrm{C}$ sous une pression d'équilibre de 0,9 bar et le concentrat s'évapore à $0^{\circ} \mathrm{C}$ sous une pression de 0,6 bar. C'est donc cette dernière pression qui règne dans l'évaporateur. Ainsi il faut comprimer la vapeur de 0,6 bar à 23,6 bars, soit un taux de compression de 39,33 (au lieu de 6,55 dans la PAC à compression classique).

Ceci nécessite l'emploi d'un compresseur à 3 étages, avec 2 refroidisseurs intermédiaires comme représenté sur la figure 7.

La puissance thermique de $10 \mathrm{~kW}$ à fournir provient alors en partie du condenseur de vapeur et en partie des deux échangeurs-refroidisseurs précédents.

Le débit d'ammoniac à évaporer et condenser est donné par la relation (voir Fig. 7) :

$$
\dot{M}_{\mathrm{vs}}=\frac{\dot{Q}_{\mathrm{us}}}{\dot{Q}_{\mathrm{c}}+\Delta H_{1}+\Delta H_{2}}=0,0065 \mathrm{~kg} / \mathrm{s} .
$$

La puissance mécanique totale à fournir aux 3 étages du compresseur est :

$$
\dot{W}_{\mathrm{s}}=\dot{M}_{\mathrm{vs}} \cdot \Delta H_{\mathrm{c}}=3,37 \mathrm{~kW}
$$




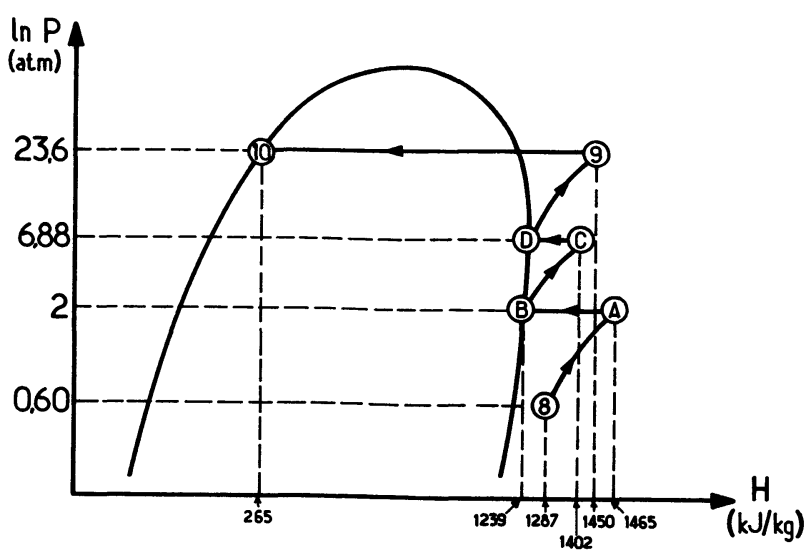

Fig. 7. - Tracé des parcours suivis par le gaz dans un compresseur tri-étagé avec deux refroidissements intermédiaires.

I. Elévation enthalpique pour le compresseur :

$1^{\text {er }}$ étage : $\Delta H_{\mathrm{c}_{1}}=H_{\mathrm{A}}-H_{8}$

$2^{\mathrm{e}}$ étage : $\Delta H_{\mathrm{c}_{2}}=H_{\mathrm{C}}-H_{\mathrm{B}}$

$3^{\mathrm{e}}$ étage : $\Delta H_{\mathrm{c}_{3}}=H_{9}-H_{\mathrm{D}}$

$\Delta H_{\mathrm{c}}=\Delta H_{\mathrm{c}_{1}}+\Delta H_{\mathrm{c}_{2}}+\Delta H_{\mathrm{c}_{3}}$.

II. Abaissement enthalpique pour les échangeurs :

$1^{\text {er }}$ refroidisseur : $\Delta H_{1}=H_{\mathrm{A}}-H_{\mathrm{B}}$

$2^{\mathrm{e}}$ refroidisseur : $\Delta H_{2}=H_{\mathrm{C}}-H_{\mathrm{D}}$

condenseur : $Q_{\mathrm{c}}=\mathrm{H}_{9}-H_{10}$

$\Delta H_{\mathrm{r}}=\Delta H_{1}+\Delta H_{2}+Q_{\mathrm{c}}$.

Débit de solvant $\dot{M}_{\mathrm{vs}}=\frac{\dot{Q}_{\mathrm{us}}}{\Delta H_{\mathrm{r}}}$.

[Operating line of the gas in the three-stage Compressor.]

ce qui correspond à un coefficient de performance de :

$$
\mathrm{COP}_{\mathrm{s}}=\frac{\dot{Q}_{\mathrm{us}}}{W_{\mathrm{s}}}=2,97
$$

D'autre part, le bilan de conservation de la matière, sur un cycle de 24 heures, en régime pseudo-stationnaire, se traduit par la relation :

$$
\dot{M}_{\mathrm{vm}} \cdot t_{\mathrm{m}}=\dot{M}_{\mathrm{vs}} \cdot t_{\mathrm{s}}
$$

où $t_{\mathrm{m}}$ et $t_{\mathrm{s}}$ sont les durées respectives de deux périodes de mélangeage et de séparation.

Le calcul donne :

$$
t_{\mathrm{m}}=9,51 \text { heures et } t_{\mathrm{s}}=14,49 \text { heures . }
$$

Finalement, le coût d'exploitation énergétique $C_{2}$ sur une période de 24 heures est :

* pendant les $t_{\mathrm{m}}$ heures de mélangeage :

$$
C_{\mathrm{m}}=0,87 \times 9,51 \times 0,5037=4,17 \mathrm{FF}
$$

* pendant les $t_{\mathrm{s}}$ heures de séparation :

$$
\begin{aligned}
& C_{\mathrm{s}}^{\prime}=3,37 \times 8 \times 0,2881=7,77 \mathrm{FF} \\
& C_{\mathrm{s}}^{\prime \prime}=3,37 \times(14,49-8) \times 0,5037=11,01 \mathrm{FF}
\end{aligned}
$$

d'où

$$
C_{\mathrm{s}} \equiv C_{\mathrm{s}}^{\prime}+C_{\mathrm{s}}^{\prime \prime}=18,78 \mathrm{FF}
$$

soit un coût total journalier :

$$
C_{2}=22,95 \mathrm{FF} / \text { jour } \text {. }
$$

Nous constatons que, dans ces conditions, l'exploitation de la PACCAFA coûte plus cher que celle d'une PAC classique. Le surcoût est de :

$$
\Delta C \equiv C_{2}-C_{1}=0,36 \mathrm{FF} / \mathrm{jour} .
$$

\section{Influence de la concentration des solutions.}

Dans le paragraphe précédent, nous avons présenté le calcul détaillé dans le cas particulier où le concentrat et le diluat avaient des compositions déterminées, soit :

$$
x_{\mathrm{c}}=0,61 \text { et } x_{\mathrm{d}}=0,54 \text {. }
$$

Nous avons refait le même calcul pour quatre valeurs du titre du concentrat :

$$
x_{\mathrm{c}}=0,65 ; 0,50 ; 0,40 \text { et } 0,30
$$

et pour divers titres du diluat comprises entre 0,05 et $\left(x_{\mathrm{c}}-0,05\right)$.

Les tableaux II, III, IV et V donnent les coûts d'exploitation $C_{2}$ avec les pourcentages de chaque composante (séparation et mélangeage) pour les divers titres $x_{\mathrm{c}}$ considérés.

La figure 8 montre, en fonction du titre de diluat $x_{\mathrm{d}}$, les valeurs du coefficient de performance, en période de séparation $\mathrm{COP}_{s}$, en période de mélangeage $\mathrm{COP}_{\mathrm{m}}$, ainsi que la valeur moyenne sur 24 heures, soit :

$$
\overline{\mathrm{COP}}=\frac{1}{24} \cdot\left(t_{\mathrm{s}} \cdot \mathrm{COP}_{\mathrm{s}}+t_{\mathrm{m}} \cdot \mathrm{COP}_{\mathrm{m}}\right)
$$

pour les 4 titres $x_{\mathrm{c}}$ étudiés.

Les figures 9 et 10 présentent respectivement pour ces mêmes titres $x_{\mathrm{c}}$, le coût d'exploitation énergétique et le surcoût d'exploitation en fonction du titre $x_{\mathrm{d}}$ du diluat.

L'examen de ces 4 tableaux et des 3 diagrammes appelle les commentaires suivants :

1. Le coefficient de performance $\overline{\mathrm{COP}}$ de la PACCAFA est généralement un peu inférieur à celui de la PAC classique $(4,58)$. Il lui devient toutefois largement supérieur pour les fortes valeurs de $x_{\mathrm{c}}$ et de $x_{\mathrm{d}}$. Par exemple on trouve $\overline{\mathrm{COP}}=6,35$ pour $x_{\mathrm{c}}=0,61$ et $x_{\mathrm{d}}=0,54$. 


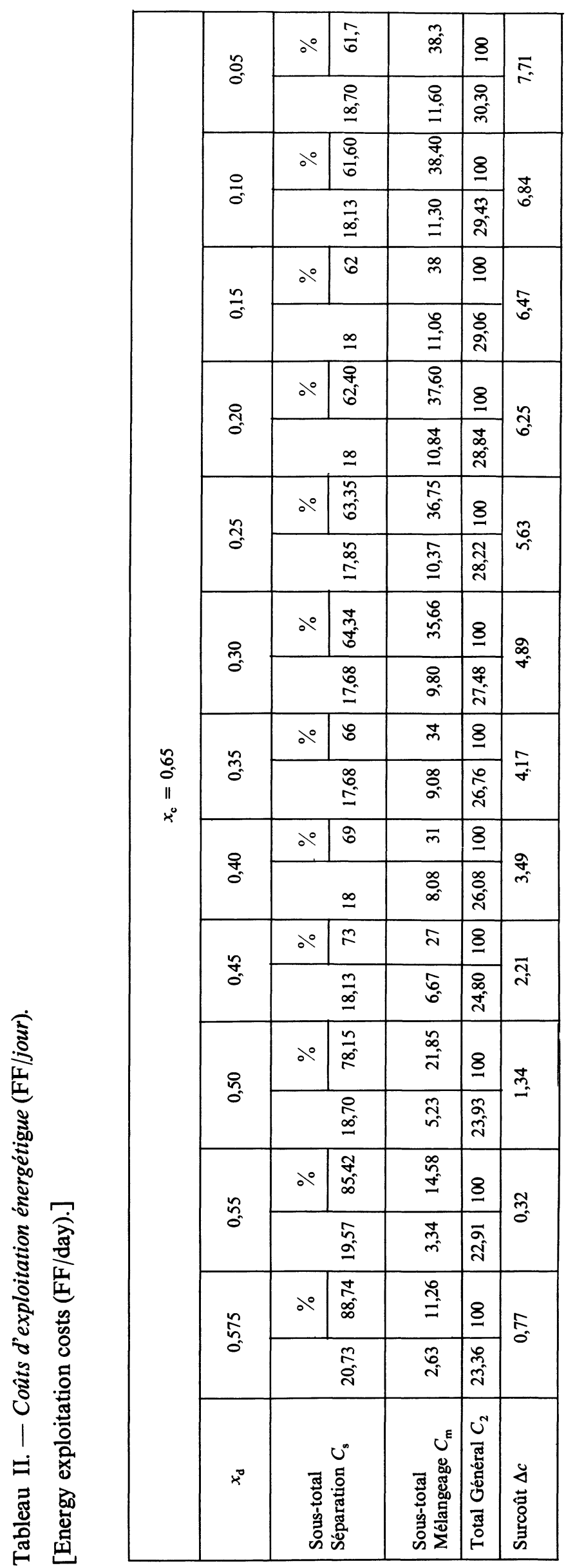


Tableau III. - Coûts d'exploitation énergétique (FF/jour).

[Energy exploitation costs (FF/day).]

\begin{tabular}{|c|c|c|c|c|c|c|c|c|c|c|c|c|c|c|c|c|c|c|}
\hline \multicolumn{19}{|c|}{$x_{\mathrm{c}}=0,50$} \\
\hline$x_{\mathrm{d}}$ & \multicolumn{2}{|c|}{0,45} & \multicolumn{2}{|c|}{0,40} & \multicolumn{2}{|c|}{0,35} & \multicolumn{2}{|c|}{0,30} & \multicolumn{2}{|c|}{0,25} & \multicolumn{2}{|c|}{0,20} & \multicolumn{2}{|c|}{0,15} & \multicolumn{2}{|c|}{0,10} & \multicolumn{2}{|c|}{0,05} \\
\hline \multirow{2}{*}{$\begin{array}{l}\text { Sous-total } \\
\text { Séparation } C_{s}\end{array}$} & \multirow[b]{2}{*}{16,21} & \multirow{2}{*}{\begin{tabular}{c|}
$\%$ \\
65,47
\end{tabular}} & \multirow[b]{2}{*}{14,03} & \multirow{2}{*}{$\begin{array}{l}\% \\
60\end{array}$} & \multirow[b]{2}{*}{13,22} & \multirow{2}{*}{$\begin{array}{l}\frac{\%}{56,57} \\
\end{array}$} & \multirow[b]{2}{*}{13,01} & $\%$ & \multirow[b]{2}{*}{12,90} & \multirow{2}{*}{\begin{tabular}{|l|}
$\%$ \\
53
\end{tabular}} & \multirow[b]{2}{*}{12,90} & $\%$ & \multirow[b]{2}{*}{12,90} & $\%$ & \multirow[b]{2}{*}{12,90} & $\%$ & \multirow[b]{2}{*}{12,90} & $\%$ \\
\hline & & & & & & & & 54,50 & & & & 52,22 & & 51,75 & & 51,27 & & 51,15 \\
\hline $\begin{array}{l}\text { Sous-total } \\
\text { Mélangeage } C_{\mathrm{m}}\end{array}$ & 8,55 & 34,53 & 9,37 & 40 & 10,15 & 43,43 & 10,86 & 45,50 & 11,40 & 47 & $\mid 11,80$ & 47,78 & 12,03 & 48,25 & 12,26 & 48,73 & 12,32 & 48,85 \\
\hline Total Général $C_{2}$ & 24,76 & 100 & 23,40 & 100 & 23,37 & 100 & 23,87 & 100 & 24,30 & 100 & 24,70 & 100 & 24,93 & 100 & 25,16 & 100 & 25,22 & 100 \\
\hline Surcoût $\Delta c$ & \multicolumn{2}{|c|}{2,17} & \multicolumn{2}{|c|}{0,81} & \multicolumn{2}{|c|}{0,78} & \multicolumn{2}{|c|}{1,28} & \multicolumn{2}{|c|}{1,71} & \multicolumn{2}{|c|}{2,11} & \multicolumn{2}{|c|}{2,34} & \multicolumn{2}{|c|}{2,57} & \multicolumn{2}{|c|}{2,63} \\
\hline
\end{tabular}

Tableau IV. - Coûts d'exploitation énergétique (FF/jour).

[Energy exploitation costs (FF/day).]

\begin{tabular}{|c|c|c|c|c|c|c|c|c|c|c|c|c|c|c|}
\hline \multicolumn{15}{|c|}{$x_{c}=0,40$} \\
\hline$x_{\mathrm{d}}$ & \multicolumn{2}{|c|}{0,35} & \multicolumn{2}{|c|}{0,30} & \multicolumn{2}{|c|}{0,25} & \multicolumn{2}{|c|}{0,20} & \multicolumn{2}{|c|}{0,15} & \multicolumn{2}{|c|}{0,10} & \multicolumn{2}{|c|}{0,05} \\
\hline \multirow{2}{*}{$\begin{array}{l}\text { Sous-total } \\
\text { Séparation } C_{s}\end{array}$} & \multirow[b]{2}{*}{12,70} & $\%$ & \multirow[b]{2}{*}{11,10} & $\%$ & \multirow[b]{2}{*}{10,60} & $\%$ & \multirow[b]{2}{*}{10,41} & $\%$ & \multirow[b]{2}{*}{10,24} & $\%$ & \multirow[b]{2}{*}{10,24} & $\%$ & \multirow[b]{2}{*}{10,24} & $\%$ \\
\hline & & 50,84 & & 48 & & 46,33 & & 45,54 & & 44,73 & & 44,38 & & 44,15 \\
\hline $\begin{array}{l}\text { Sous-total } \\
\text { Mélangeage } C_{\mathrm{m}}\end{array}$ & 12,28 & 49,16 & 12,04 & 52 & 12,28 & 53,67 & 12,50 & 54,56 & 12,65 & 55,27 & 12,83 & 55,62 & 12,95 & 55,85 \\
\hline Total Général $C_{2}$ & 24,98 & 100 & 23,14 & 100 & 22,88 & 100 & 22,91 & 100 & 22,89 & 100 & 23,07 & 100 & 23,19 & 100 \\
\hline Surcoût $\Delta c$ & \multicolumn{2}{|c|}{2,39} & \multicolumn{2}{|c|}{0,55} & \multicolumn{2}{|c|}{0,29} & \multicolumn{2}{|c|}{0,32} & \multicolumn{2}{|c|}{0,30} & \multicolumn{2}{|c|}{0,48} & \multicolumn{2}{|c|}{0,60} \\
\hline
\end{tabular}

Tableau V. - Coûts d'exploitation énergétique $(\mathrm{FF} / j o u r)$.

[Energy exploitation costs (FF/day).]

\begin{tabular}{|c|c|c|c|c|c|c|c|c|c|c|}
\hline \multicolumn{11}{|c|}{$x_{c}=0,30$} \\
\hline$x_{\mathrm{d}}$ & \multicolumn{2}{|c|}{0,25} & \multicolumn{2}{|c|}{0,20} & \multicolumn{2}{|c|}{0,15} & \multicolumn{2}{|c|}{0,10} & \multicolumn{2}{|c|}{0,05} \\
\hline \multirow{2}{*}{$\begin{array}{l}\text { Sous-total } \\
\text { Séparation } C_{\mathrm{s}}\end{array}$} & \multirow[b]{2}{*}{11,54} & $\%$ & \multirow[b]{2}{*}{10,33} & $\%$ & \multirow[b]{2}{*}{9,94} & $\%$ & \multirow[b]{2}{*}{9,85} & $\%$ & \multirow[b]{2}{*}{9,67} & $\%$ \\
\hline & & 42,74 & & 44 & & 43,25 & & 42,86 & & 42,43 \\
\hline $\begin{array}{l}\text { Sous-total } \\
\text { Mélangeage } C_{\mathrm{m}}\end{array}$ & 15,46 & 57,27 & 13,11 & 56 & 13,04 & 56,75 & 13,13 & 57,14 & 13,12 & 57,57 \\
\hline Total général $C_{2}$ & 27 & 100 & 23,44 & 100 & 22,98 & 100 & 22,98 & 100 & 22,79 & 100 \\
\hline Surcoût $\Delta c$ & \multicolumn{2}{|c|}{4,41} & \multicolumn{2}{|c|}{0,85} & \multicolumn{2}{|c|}{0,39} & \multicolumn{2}{|c|}{0,39} & \multicolumn{2}{|c|}{0,20} \\
\hline
\end{tabular}




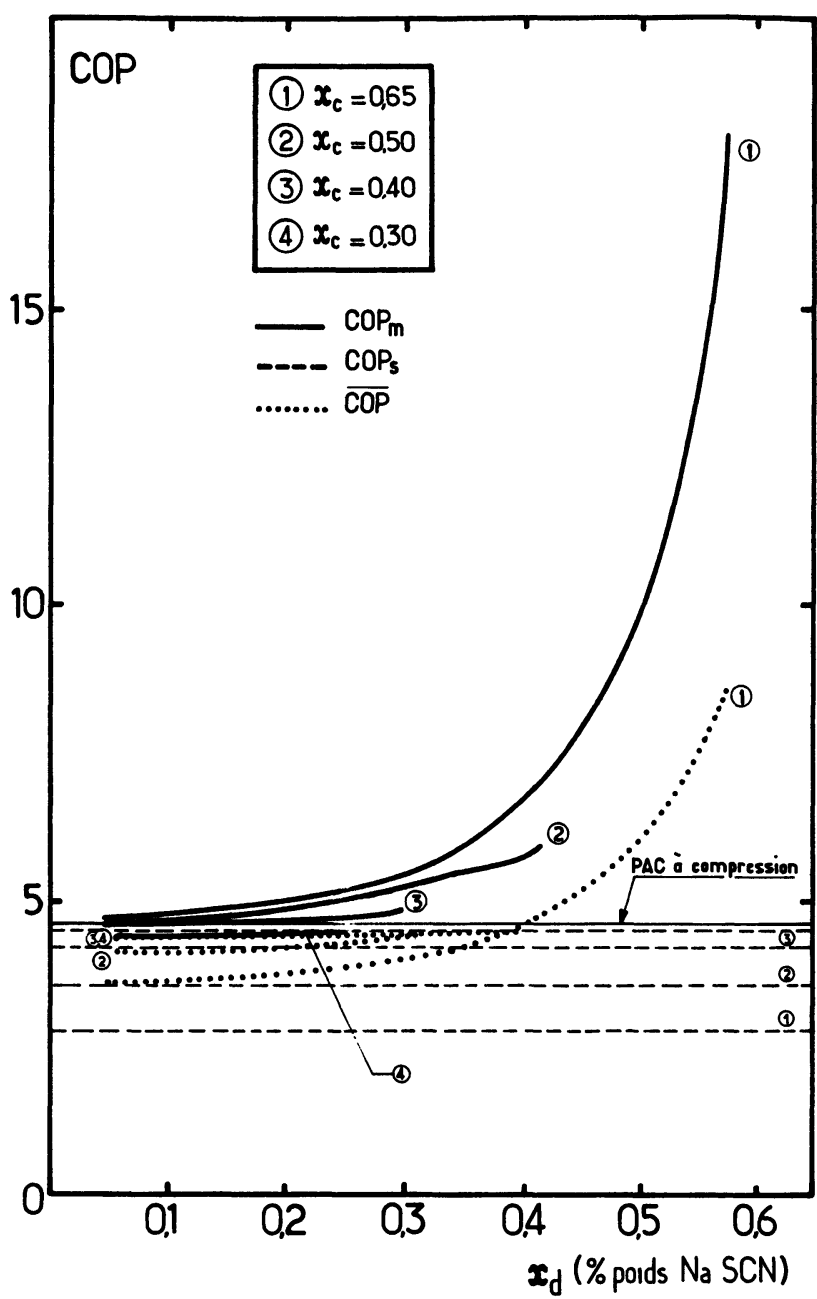

Fig. 8. - Coefficient de performance en fonction du titre de diluat, pour 4 valeurs du titre du concentrat.

[Coefficient of performance as a function of the diluate concentration for 4 concentrate concentrations.]

2. Le coût total d'exploitation de la PACCAFA est toujours supérieur à celui de la PAC classique. Toutefois le surcoût est très faible dans les cas où $x_{\mathrm{c}}$ et $x_{\mathrm{d}}$ sont tous deux faibles. Par exemple pour $x_{\mathrm{c}}=0,40$, le surcoût reste inférieur à $0,32 \mathrm{FF} /$ jour (soit $1,4 \%$ du coût total) quand $x_{\mathrm{d}}$ est compris entre 0,15 et 0,25 .

3. Il est important de remarquer que ce fort coût d'exploitation de la PACCAFA est dû, pour une grande part, au fait que la durée $t_{\mathrm{s}}$ de séparation est nettement supérieure à la durée des heures creuses $\left(t_{\mathrm{c}}=8\right.$ heures $)$. Il en résulte qu'une fraction non négligeable de la surconsommation d'énergie en régime de séparation doit être payée au tarif fort des heures pleines.

\section{Recherche des conditions où la PACCAFA serait plus économique.}

Recherchons quelles devraient être les conditions tarifaires de l'électricité pour que le coût d'exploita-

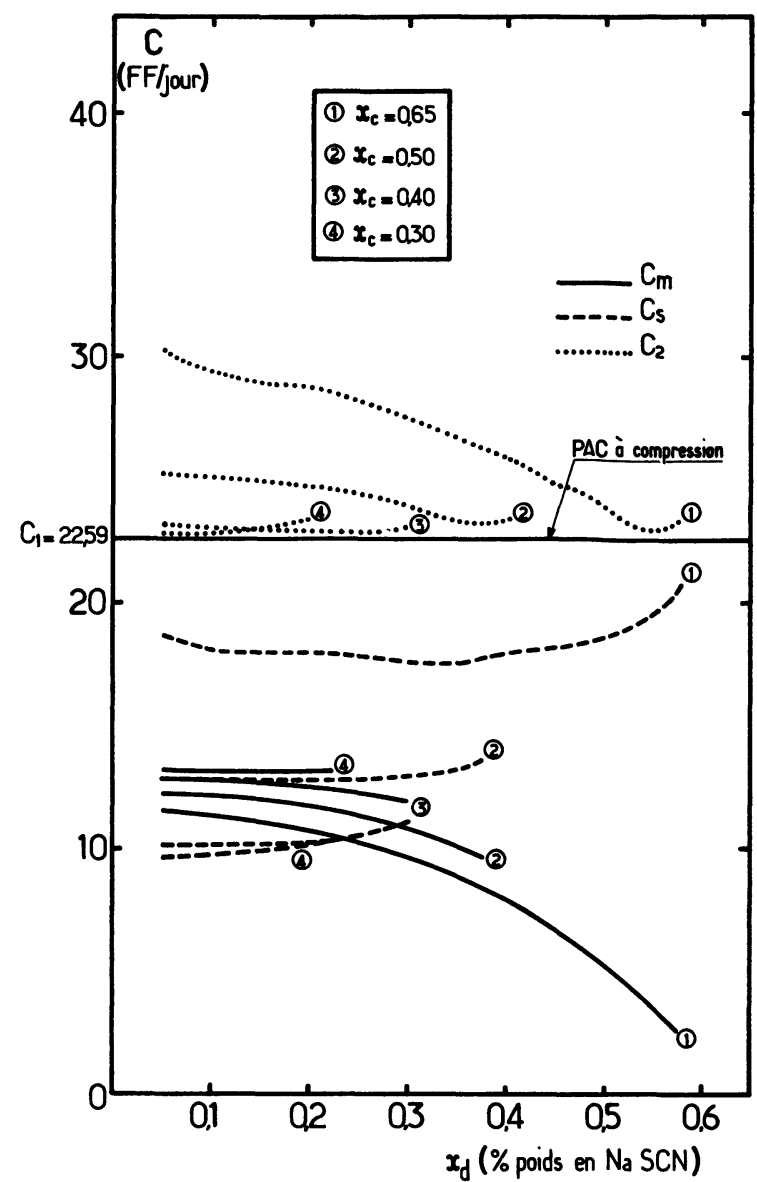

Fig. 9. - Coûts d'exploitation pour une puissance utile de $10 \mathrm{~kW}$, en fonction du titre du diluat, pour 4 valeurs du titre du concentrat.

[Exploitation costs for a useful power $=10 \mathrm{~kW}$ as a function of diluate concentration, for 4 concentrate concentrations.]

tion de la PACCAFA devienne inférieur à celui d'une PAC classique.

6.1 DuRÉE Minimale DE LA PÉRIOde « HEURES CREUSES ». - Supposons dans un premier scénario, que les prix de vente de l'électricité restent les mêmes, soit $0,2881 \mathrm{~F}$ en heures creuses et $0,5037 \mathrm{FF} / \mathrm{kWh}$ en heures pleines et calculons quelle devrait être la durée minimale de la période « heures creuses » pour réaliser l'inégalité :

$$
C_{2} \leqslant C_{1} .
$$

Le résultat du calcul est :

$$
t_{\mathrm{c}} \geqslant \frac{\left(\dot{W}_{\mathrm{m}} \cdot t_{\mathrm{m}}+\dot{W}_{\mathrm{s}} \cdot t_{\mathrm{s}}-24 \cdot \dot{W}\right) \cdot v_{\mathrm{p}}}{\left(\dot{W}_{\mathrm{s}}-\dot{W}\right) \cdot\left(v_{\mathrm{p}}-v_{\mathrm{c}}\right)} .
$$

La figure 11 montre les valeurs minimales de $t_{\mathrm{c}}$ en fonction de $x_{\mathrm{c}}$ et de $x_{\mathrm{d}}$. 


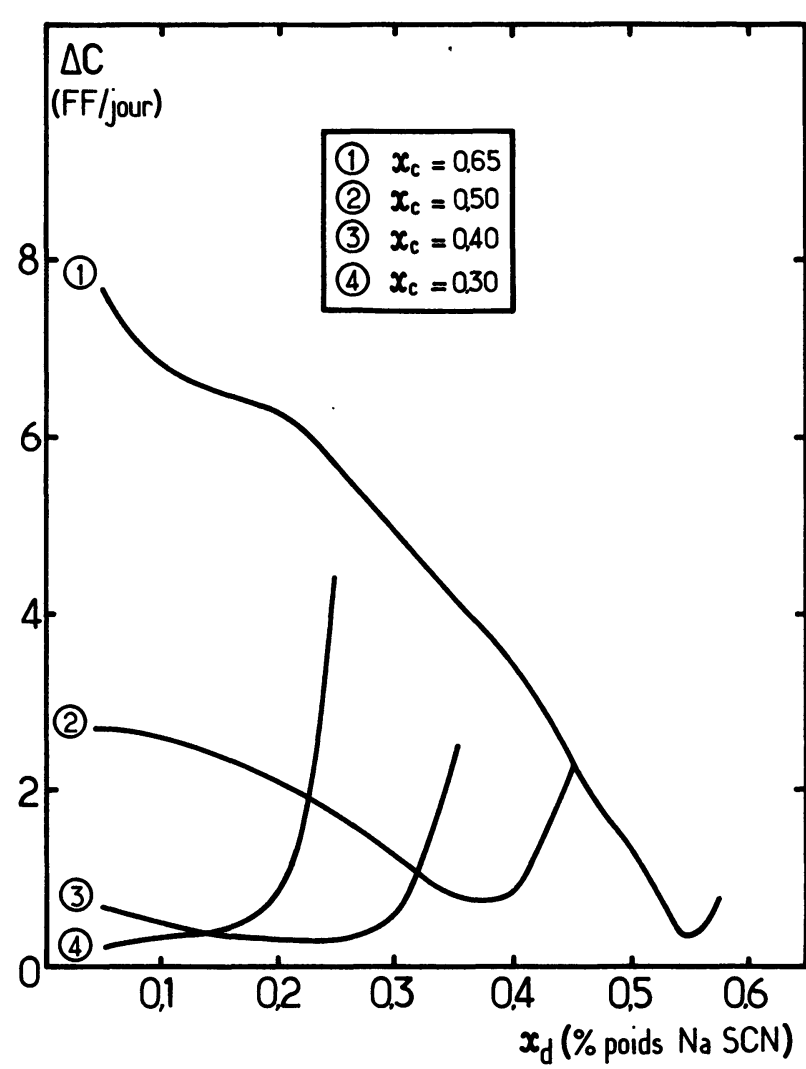

Fig. 10. - Surcoût d'exploitation énergétique pour une puissance utile de $10 \mathrm{~kW}$, en fonction du titre $x_{\mathrm{d}}$ du diluat, pour 4 valeurs du titre du concentrat.

[Exploitation Extra-Cost for a useful power $=10 \mathrm{~kW}$ as a function of diluate concentration for 4 concentrate concentrations.]

Nous constatons que toutes les valeurs de la durée d'heures creuses sont nettement supérieures à la valeur actuelle de $t_{\mathrm{c}}$, soit 8 heures en particulier pour les faibles valeurs de $x_{\mathrm{c}}$ et $x_{\mathrm{d}}$.

La seule valeur de $t_{\mathrm{c}}$ qui se rapproche de la valeur actuelle correspond au cas suivant : $x_{\mathrm{c}}=0,65$ et $x_{\mathrm{d}}=0,55$.

6.2 MOdUlATION MINIMALE DU PRIX DE L'ÉLECTRICITÉ. - Supposons maintenant dans un deuxième scénario que les périodes d'heures pleines et d'heures creuses gardent les mêmes durées, soit 16 heures et 8 heures, mais que le rapport des prix de vente de l'électricité pendant ces périodes soit modifié, pour obtenir à nouveau l'inégalité.:

$$
C_{2} \leqslant C_{1}
$$

Soit $k \equiv v_{\mathrm{p}} / v_{\mathrm{c}}$ le rapport de ces prix. Le résultat du calcul est :

$$
k \geqslant \frac{t_{\mathrm{c}}\left(\dot{W}_{\mathrm{s}}-\dot{W}\right)}{t_{\mathrm{p}} \cdot \dot{W}-t_{\mathrm{m}} \cdot \dot{W}_{\mathrm{m}}-\left(t_{\mathrm{s}}-t_{\mathrm{c}}\right) \dot{W}_{\mathrm{s}}} .
$$

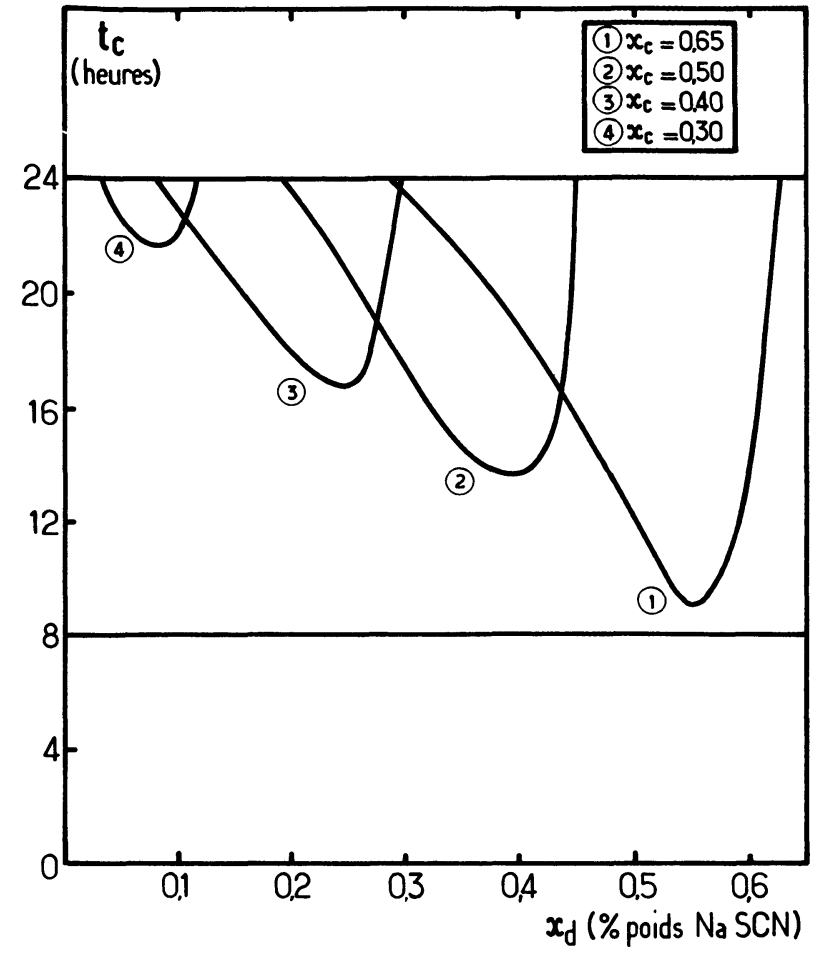

Fig. 11. - Durée minimale de la période « heures creuses » pour que la PACCAFA soit plus économique qu'une PAC classique.

[The minimum duration of an « off-peak period " to the CAHPW would be smaller than that of a standard Compression Heat Pump.]

Les valeurs numériques du rapport $k$ sont données dans le tableau VI pour diverses valeurs de $x_{c}$ et $x_{\mathrm{d}}$.

Rappelons que la valeur actuelle de $k$ est :

$$
k=0,5037 / 0,2881=1,75 .
$$

Le tableau VI montre que le rapport $k$ prend des valeurs très fortes (irréalistes) pour les faibles valeurs de $x_{\mathrm{c}}$ et de $x_{\mathrm{d}}$.

Les seules valeurs de $k$ qui ne semblent pas trop éloignées de la valeur actuelle correspondent à de très fortes valeurs de $x_{\mathrm{c}}$ et de $x_{\mathrm{d}}$.

Par exemple, on obtient $k \geqslant 1,95$ pour $x_{\mathrm{c}}=0,65$ et $x_{\mathrm{d}}=0,55$.

Supposons à titre d'exemple, que le rapport $k$ prenne la valeur 2,5 ce qui assurerait une rentabilité certaine à la PACCAFA, comparée à la PAC. Pour que le prix moyen du $\mathrm{kWh}$ sur $24 \mathrm{~h}$ reste le même, il faudrait que les prix de vente deviennent les suivants :

* en heures creuses : $v_{\mathrm{c}}=0,2159$ (au lieu de $0,2881)$

* en heures pleines : $v_{\mathrm{p}}=0,5398$ (au lieu de 0,5037). 
Tableau VI. - Valeurs du coefficient de proportionalité $k$.

[Values of the proportionality coefficient $k$.]

\begin{tabular}{|c|c|c|c|c|c|}
\hline \multirow{13}{*}{ 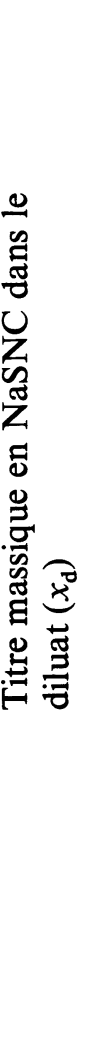 } & 0,575 & 2,26 & & & \\
\hline & 0,55 & 1,95 & & & \\
\hline & 0,50 & 2,88 & & & \\
\hline & 0,45 & 2,95 & 3,25 & & \\
\hline & 0,40 & 3,10 & 3,68 & & \\
\hline & 0,35 & 3,50 & 3,87 & 4,10 & \\
\hline & 0,30 & 3,85 & 5,60 & 6,50 & \\
\hline & 0,25 & 4,15 & 6,85 & 8,50 & 9,10 \\
\hline & 0,20 & 4,35 & 7,60 & 12,10 & 13 \\
\hline & 0,15 & 4,50 & 8 & 13,56 & 14,10 \\
\hline & 0,10 & 5,20 & 8,40 & 14 & 16,30 \\
\hline & 0,05 & 5,45 & 9,95 & 15,8 & 21 \\
\hline & & 0,65 & 0,50 & 00,40 & 0,30 \\
\hline
\end{tabular}

Titre massique en NaSCN dans le concentrat $\left(x_{c}\right)$.

\section{Conclusion.}

Dans les conditions tarifaires actuelles de l'électricité vendue par E.D.F., le nouveau système de pompe à chaleur à compression-absorption, envisagé ici, n'a pas un coût d'exploitation inférieur à celui d'une pompe à chaleur à compression classique. Pour qu'il le devienne, il faudrait:

* ou bien que la période journalière à faible tarif (heures creuses) soit allongée (de 8 heures actuelles) à environ 16 heures ;

* ou bien que le rapport des prix de vente de l'électricité en heures pleines et en heures creuses soit porté de 1,75 (valeur actuelle) à environ 2,5.

Rappelons enfin que nous n'avons comparé dans le présent rapport que les coûts d'exploitation des deux types de pompes à chaleur. Or le coût d'investissement de la PACCAFA est sans aucun doute nettement supérieur à celui d'une PAC classique, du fait même de la présence des réservoirs de stockage et de la plus grande complexité des circuits. Pour compenser ce surcoût d'investissement, il faudrait donc que l'économie sur les coûts d'exploitation soit encore plus grande que celle envisagée ici.

Il est peu probable que de telles conditions puissent jamais être remplies.

\section{References}

[1] Dietrich, E. et Le Goff, P., Rev. Phys. Appl. 21 (1986) 45-52.

[2] Dietrich, E., Barkaoui, M. et Le Goff, P., Entropie 118 (1984) 8-12.

[3] Trap, J. C., Dietrich, E. et Le Goff, P., Proceedings of the International workshop on « Heat Transformation and Storage » C.E.E., ISPRA, Oct. 1985.

[4] BERnier, J., La pompe à chaleur: mode d'emploi, tomes 1 et 2 (Editions Pyc, Paris) 1979.
[5] Infante Ferreira, C. A., Solar Energy 32 (1984) 231-236.

[6] Tables of thermodynamic properties of ammonia. Circular of the bureau of standards, $n^{\circ} 142$, Avril 1923, Washington.

[7] La nouvelle tarification des fournitures d'électricité, catalogue E.D.F. (direction de la distribution) 1984. 